SECCIÓN ABIERTA 

REVISTA DE DERECHO UNED, NÚM. 8, 2011

\title{
DERECHOS FUNDAMENTALES Y VIOLENCIA DE GÉNERO
}

\author{
Jorge Alguacil GonZÁlez-Aurioles \\ Profesor Contratado Doctor de Derecho Constitucional
}

Sumario: 1. Introducción: derechos fundamentales y violencia de género.- 2. La constitucionalidad de la ley integral contra la violencia de género: igualdad y Constitución.- 3. Incidencia de la ley en la sociedad.- 4. A modo de conclusiones: la igualdad como imperativo constitucional.

\section{INTRODUCCIÓN: DERECHOS FUNDAMENTALES Y VIOLENCIA DE GÉNERO}

Hay ciertos artículos de nuestra Carta Magna que abren nuestra sociedad a la transformación. Uno de ellos es el 9.2: «corresponde a los poderes públicos promover las condiciones para que la libertad y la igualdad del individuo y de los grupos en que se integra sean reales y efectivas; remover los obstáculos que impidan o dificulten su plenitud y facilitar la participación de todos los ciudadanos en la vida política, económica, cultural y social». Recogido en el Título Preliminar de la Constitución, donde se encuentran los grandes principios políticos y decisiones fundamentales que iluminan todo el texto constitucional, supone, como observa Garrorena Morales, reconocer de forma frontal y generalizada «la constitutiva desigualdad e injusticia que afecta al sistema capitalista, lo cual tiene ya un delicado y contradictorio aire de denuncia del sistema mismo» ${ }^{1}$. Implica, en defini-

${ }^{1}$ Garrorena Morales, Á, El Estado español como Estado social y democrático de Derecho, Madrid: Tecnos, 1992, pág. 59. 
tiva, un reconocimiento de la existencia de incorrecciones en la sociedad y de la necesidad, en consecuencia, de superarlas por medio de la actuación de los poderes públicos.

Quizá por eso, de Otto entiende posible, en virtud de este precepto, realizar una política de derechos fundamentales encaminada a asegurar la libertad y la igualdad de todos. La cláusula del Estado social (artículo 1.1 CE) impone una política de los derechos fundamentales que sirva para generalizar y socializar estos derechos ${ }^{2}$, para universalizar, en fin para democratizar el disfrute de los derechos. Tal política ha de realizarse a través del poder público principalmente llamado a hacer efectivos los derechos, el legislador. Aquéllos, como es conocido, no son sólo derechos subjetivos, son también principios objetivos que informan todo el ordenamiento jurídico. Esta dimensión objetiva de los derechos impone a todos los poderes públicos la tarea de asegurarlos, el papel del legislador ha de ser especialmente activo. El legislador pasa, pues, de ser un «enemigo potencial» de la Constitución y de los derechos fundamentales a ser el poder público indispensable que necesitan para desplegar plena eficacia, su relación con la Carta Magna y con los derechos fundamentales adquiere una nueva dimensión: la cooperativa. Ambos, derechos y ley, deben cooperar para asegurar la fuerza normativa de aquéllos. Esto fue reconocido inicialmente en Alemania, por ejemplo, por Hesse ${ }^{3}$ y su doctrina se proyectó, entre otros, entre sus discípulos más inmediatos, Hollerbach o Häberle. Para Häberle, por ejemplo, a la doble dimensión de los derechos fundamentales, subjetiva y objetiva, le corresponde una doble función del legislador, como limitador y como configurador de los derechos fundamentales ${ }^{4}$.

La Ley Integral de Medidas contra la Violencia de Género podría orientarse a realizar lo planteado, en fin, quizá puede ser ejemplo del legislador al que apela Häberle, configurador de los derechos fundamentales. Seguramente los derechos a la vida, al libre desarrollo de la personalidad, a la libertad personal, al honor, en fin, a la libertad de pensamiento, a la libertad de expresión y tantas otras libertades, difí-

${ }^{2}$ La cláusula del Estado social sirve para generalizar y socializar los derechos de libertad, De Otto, I, «La regulación del ejercicio de los derechos y libertades. La garantía de su contenido esencial en el artículo 53.1 de la Constitución», en Luis Martín Retortillo, Ignacio de Otto y Pardo, Derechos fundamentales y Constitución, Madrid, Cívitas, 1988, págs. $165-169$.

${ }^{3}$ Hesse, Grundzüge des Verfassungsrechts der Bundesrepublick Deutschland, Karlsruhe, CF Müller, $1^{\text {a }}$ ed. 1967, pág. 137.

${ }^{4}$ HäBerle, Die Wesensgehaltsgarantie des Artikel 19 Abs 2 Grundgesetz, Karlsruhe, op. cit, págs. 4 y ss y 181 y ss. (en la versión catellana, 7 y ss, 175 y ss.). 
cilmente pueden ser disfrutados por las víctimas de situaciones de violencia y vejación en el seno de una relación afectiva. La necesidad de remover los obstáculos que impiden que la libertad y la igualdad, también de las personas víctimas de esta situación, sea real y efectiva, podría estar en el espíritu de la ley; y ello la convertiría en una norma dotada de un especial valor, el de contribuir a hacer efectivos los derechos en nuestra actual sociedad. En fin, a hacer viable una política de derechos fundamentales orientada a superar un modelo de sociedad, anclado en ciertas desigualdades lo que sin duda alguna podría crear márgenes al programa emancipatorio de nuestra Carta Magna.

\section{LA CONSTITUCIONALIDAD DE LA LEY: IGUALDAD Y CONSTITUCIÓN}

De la exposición de motivos de la ley cabe extraer tres claras directrices. En primer lugar, mediante esta norma jurídica se pretende expulsar definitivamente la violencia de género del ámbito privado, al conferirla una dimensión pública. Se trata «del símbolo más brutal de la existencia de desigualdad en nuestra sociedad» y ello necesariamente ha de interpelar a todos los poderes públicos y privados de la sociedad avanzada de la que habla el preámbulo de nuestra Carta Magna. En segundo término, el preámbulo de la ley identifica precisamente esta violencia como un ataque a los derechos fundamentales más básicos de las personas que lo sufren, convierte el problema existente y las posibles respuestas al mismo, en una cuestión de política de derechos fundamentales. La obligación de adoptar medidas de acción positiva para hacer reales y efectivos los derechos de estas personas, con cita expresa del artículo 9.2 CE, justifica la existencia de la ley. Y ello la conecta directamente con la política de derechos de la que nos hablara de Otto. En último lugar, atendiendo a las recomendaciones de los organismos internacionales, la respuesta a esta violación de los derechos de las mujeres se configura como interdisciplinar e integral, si bien se dota de un especial énfasis al proceso de socialización y educación. Los obstáculos que impiden que la libertad y la igualdad de las mujeres sean reales y efectivas no pueden permanecer por más tiempo en el ámbito privado. Si realmente se quieren remover tales obstáculos han de poder ser elevados, en primer lugar, a la esfera de lo público. Por otra parte, la remoción de tales obstáculos, impuesta por la Constitución en su artículo 9.2 CE, puede y debe ser realizada por el legislador, mediante el establecimiento de medidas que de una forma integral traten de superar esta situación 
de la mujer y favorezcan posibilidades de alcanzar en nuestras actuales sociedades cotas mayores de igualdad.

Nos encontramos, pues, ante una norma jurídica que integra políticas promocionales de la mujer, insertas en esta vocación transformadora de la Constitución, reflejada en su artículo 9.2 CE. Esta dimensión emancipadora de nuestra Carta Magna se puede proyectar en diferentes aspectos; también en la promoción del sexo femenino como perteneciente a un grupo históricamente discriminado. La ley integral contra la violencia de género parecía tener en su origen una primera finalidad, de la mayor importancia: contribuir a superar una sociedad que podía encubrir o hacer más justificables ciertos delitos en el seno de las relaciones afectivas de pareja, por una renovada comunidad democrática, que regida por el principio de igualdad, hiciera imposible tales atentados. Los instrumentos de que se pertrechó fueron diversos: la educación, sin duda alguna el instrumento más precioso de que dispone el Estado para asegurar la igualdad en una sociedad, constituyó uno de los pilares de la ley. Esta norma jurídica insiste en que la formación en el respeto a la igualdad entre hombres y mujeres debe ser uno de los fines del sistema educativo español. Tal principio de igualdad se transmitirá desde la educación infantil hasta el bachillerato: la Educación Infantil contribuirá a desarrollar en la infancia el aprendizaje en la resolución pacífica de conflictos, la primaria a desarrollar en el alumnado su capacidad para adquirir habilidades en la resolución pacífica de conflictos y para comprender y respetar la igualdad entre sexos. La Educación Secundaria Obligatoria contribuirá a desarrollar en el alumnado la capacidad para relacionarse con los demás de forma pacífica y para conocer, valorar y respetar la igualdad de oportunidades de hombres y mujeres, y el Bachillerato y la Formación Profesional contribuirán a desarrollar en el alumnado la capacidad para consolidar su madurez personal, social y moral, que les permita actuar de forma responsable y autónoma y para analizar y valorar críticamente las desigualdades de sexo y fomentar la igualdad real y efectiva entre hombres y mujeres. La enseñanza para las personas adultas incluirá entre sus objetivos desarrollar actividades en la resolución pacífica de conflictos y fomentar el respeto a la dignidad de las personas y a la igualdad entre hombres y mujeres. Las Universidades incluirán y fomentarán en todos los ámbitos académicos la formación, docencia e investigación en igualdad de género y no discriminación de forma transversal. Por lo demás, la Administración tendrá que revisar los materiales y libros de texto para garantizar la ausencia de conceptos o referencias que puedan fomentar la desigualdad. Por último, en el Consejo Es- 
colar tendrán presencia las organizaciones de mujeres, el Instituto de la Mujer y expertos reconocidos en la lucha contra la violencia por razones de sexo. También se crean derechos específicos de las mujeres víctimas de la violencia de género, medidas de protección en el ámbito social, medidas de apoyo económico, se crea un sistema de tutela de estas mujeres a través de unos juzgados específicos, en fin, se incrementan las penas para las personas que cometan delitos o faltas que puedan tipificarse como de violencia de género.

Mucho se ha escrito sobre la conveniencia de estas medidas educativas, pero también acerca de las dudas de constitucionalidad que despertó inicialmente la incorporación de estas medidas de discriminación positiva en un campo tan sensible como el del Derecho Penal. En concreto, se incorpora un título, el IV, relativo a la tutela penal, que por primera vez eleva el género a criterio cualificador de la conducta delictiva y, consiguientemente, del agravamiento de la pena. La tramitación parlamentaria de la ley ya nos alertó de cómo era este extremo el que provocaba mayores discusiones. El sexo de la víctima y con ello del sujeto activo cualificaba la conducta delictiva. Por primera vez se introducía un elemento discriminatorio a favor de la mujer en un campo tan sensible como el del Derecho Penal ${ }^{5}$, aunque ello motivó quizá uno de los pocos cambios en la tramitación de la ley: se incluyó una cláusula que permitía extender esta agravación de las penas a aquella persona especialmente vulnerable que conviva con el autor. En cualquier caso, se llegó incluso a alertar de que la inserción de este Título IV podía deslegitimar las iniciales buenas intenciones de la ley. Ciertamente hubo más de una voz discrepante que defendió la ley en su totalidad, aunque las sucesivas sentencias del Tribunal Constitucional sobre el particular vinieron a disipar al menos la legitimidad de la norma.

En efecto, nuestro supremo intérprete constitucional, en su conocida resolución 12/2008, de 29 de enero, vino a confirmar los iniciales razonamientos que defendían la constitucionalidad de la ley. Ésta se sustenta en dos premisas: en primer lugar, porque a juicio de nuestro supremo intérprete constitucional, y siguiendo una amplia jurisprudencia, la política criminal depende del legislador, el Tribunal

${ }^{5}$ En cualquier caso, era diversa la forma de establecer tal discriminación en los preceptos mencionados: las posibilidades oscilaban entre introducir un tipo cualificado del tipo general en función del sexo de la víctima (protección contra las lesiones) o convertir el sexo de la víctima en criterio para transformar lo que en supuestos ordinarios era una falta en un delito (protección contra malos tratos, protección contra las amenazas de un mal que no constituya delito, coacciones). 
Constitucional sólo puede realizar un control de mínimos: a juicio del tribunal, «nuestro análisis actual del art. 153.1 CP no puede serlo de su eficacia o de su bondad, ni alcanzar a calibrar el grado de desvalor de su comportamiento típico o el de severidad de su sanción. Sólo nos compete enjuiciar si se han respetado los límites externos que el principio de igualdad impone desde la Constitución a la intervención legislativa ${ }^{6}$. Pero en cualquier caso, la principal objeción del cambio legislativo, la supuesta creación de un Derecho penal de autor, queda desbaratada con la «adición del artículo de la persona especialmente vulnerable que conviva con el autor como posible sujeto del delito»; la diferencia introducida por la ley, a juicio del Tribunal, es razonable, fruto de la amplia libertad de opción de que goza el legislador penal, «que, por la limitación y la flexibilidad de sus previsiones punitivas, no conduce a consecuencias desproporcionadas». Se trata, en opinión de nuestro supremo intérprete constitucional, "de una diferenciación razonable porque persigue incrementar la protección de la integridad física, psíquica y moral de las mujeres en un ámbito, el de la pareja, en el que están insuficientemente protegidos, y porque persigue esta legítima finalidad de un modo adecuado a partir de la, a su vez, razonable constatación de una mayor gravedad de las conductas diferenciadas, que toma en cuenta su significado social objetivo y su lesividad peculiar para la seguridad, la libertad y la dignidad de las mujeres» ${ }^{7}$.

Lo cierto, así, es que esta igualdad no implica la represión de ningún sujeto, sino la protección especial de un colectivo que está absolutamente necesitado de ello. Si bien el incremento de las penas no revierte en una disminución de los delitos, sí incide de forma decisiva en lo que denomina "prevención general positiva ${ }^{8}$. La nueva tutela penal puede entenderse como una «acción positiva» o una "discriminación positiva» reconocida expresamente en el ordenamiento jurídico comunitario y español. En definitiva, la intervención punitiva puede responder a una mayor preocupación frente a determinadas conductas discriminatorias en determinados contextos. Al igual que el rechazo a la violencia contra extranjeros motivada por causas racistas o xenófobas ha conducido a incrementar las penas en este ámbito, también podría justificarse la represión penal de la violencia de género. Un antecedente está, pues, en la agravante general de discrimi-

${ }^{6}$ STC 59/2008, de 14 de mayo F.J. 6

${ }^{7}$ STC 59/2008, de 14 de mayo, FJ 12

${ }^{8}$ Esta fue la postura defendida, por ejemplo, por PECES-BARBA, G, »Intervención», Diario de sesiones del Congreso de los Diputados. Comisión de Trabajo y Asuntos Sociales, $n^{\circ} 64$, VII Legislatura, año 2004, pág. 10 
nación del artículo $224.4^{\circ}$ del Código Penal, que sólo será apreciable cuando el delito se cometa por alguno de los motivos discriminatorios que en ella se contemplan expresamente: «racistas, antisemitas u otra clase de discriminación referente a la ideología, religión o creencias de la víctima, la etnia, la raza o la nación a la que pertenezca, su sexo, su orientación sexual, o la enfermedad o minusvalía». El incremento de la pena podría estar justificada por la mayor reprochabilidad del hecho punible; pero estaríamos ante una circunstancia modificativa de la responsabilidad de naturaleza subjetiva. Los problemas de prueba podrían ser considerables, pero la norma tiende a reforzar la vigencia social de los valores constitucionalizados. Quizá pudiera emplearse en este caso criterios semejantes. Para evitar la acusación de estar retomando un Derecho Penal de autor cabría suprimir la necesaria condición de sujeto activo al varón, dejando como sujeto pasivo a la mujer. En los actos de violencia xenófoba o racista no se tipifica al sujeto activo, se incide simplemente en el tipo mate$\operatorname{rial}^{9}$ (incluso hubiera cabido tipificar el sujeto pasivo como el inmigrante, y esto hubiera incidido en la denominada prevención general positiva). Al quedar indeterminado el sujeto activo no subsisten reparos de inconstitucionalidad. La solución definitivamente adoptada, que incluye una cláusula de apertura (si la víctima fuera una persona especialmente vulnerable que conviva con el autor), permite extender el sujeto activo a otros sujetos del ámbito doméstico además del marido. Si a ello unimos que de la redacción literal de los preceptos en cuestión se desprende exclusivamente la posibilidad, que no la obligación, de castigar las conductas tipificadas con la agravación de la pena prevista, podríamos convenir que la objeción esgrimida está superada.

\section{INCIDENCIA DE LA LEY EN LA SOCIEDAD}

Como es conocido, la capacidad normativa de lo que usualmente conocemos como Constitución depende, en palabras de Lasalle, de su coincidencia con la constitución real. Donde esto no ocurre se produce un conflicto inevitable en el que, a la larga, la constitución

${ }^{9}$ Artículo 510.1 del Código Penal: »Los que provocaren a la discriminación, al odio o a la violencia contra grupos o asociaciones, por motivos racistas, antisemitas u otros referentes a ideología, religión o creencias, situación familiar, la pertenencia de sus miembros a una etnia o raza, su origen nacional, su sexo, orientación sexual, enfermedad o minusvalía, serán castigados con la pena de prisión de uno a tres años y multa de seis a doce meses» 
escrita, que es en estos casos para el autor alemán una simple hoja de papel, sucumbe ante las efectivas relaciones de poder existentes. Ya puede haber democratizado nuestra Carta Magna los derechos, y haberlos extendido a toda la sociedad, también a las mujeres. Bien puede nuestra Constitución haber proclamado la igualdad como un valor (artículo 1.1 CE), como un derecho fundamental (artículo 14 $\mathrm{CE}$ ) e incluso como un principio político promocional de los poderes públicos (artículo 9.2 CE). Si tal democratización de los derechos y tal extensión de la igualdad no se inserta en las relaciones efectivas de poder, si no se subordinan las mismas al logro de aquéllas; si, en fin, no se logra impregnar la sociedad del espíritu constitucional necesario para hacer efectivo tales principios, de poco puede servir lo preceptuado por la Constitución ${ }^{10}$. La Ley integral de medidas contra la violencia de género parece orientarse en aras del logro de estos objetivos. De los debates parlamentarios, de su preámbulo, en fin, de su muy importante capítulo sobre educación, sólo cabe corroborar esta inicial afirmación. En cualquier caso, cumple ahora, tras siete años de su entrada en vigor, analizar la efectiva realización de tales cometidos.

Cabe señalar, en primer lugar, dos datos, que quizá puedan ser indicativos de un cierto cambio de tendencia en esta lacra social: por un lado, el número de mujeres muertas a manos de sus parejas o exparejas ha descendido de 69 en 2004 (año de entrada en vigor de la ley) a 55 en 2009. Si bien este año ha habido un muy inquietante repunte del número de mujeres asesinadas por sus parejas, podemos destacar con orgullo que España está por debajo de de la media europea y mundial en muertes por violencia de género. Por otro, nunca en la historia constitucional han existido tantos mecanismos de lucha, efectivamente activos, contra esta lacra social: la creación de unos juzgados especiales de Violencia de Género ha podido ofrecer protección específica a las mujeres maltratadas. Sólo en el cuarto trimestre de 2009 se tramitaron 10.226 órdenes de protección y en el primer trimestre de 2009 se celebraron más de 2.460 juicios de faltas relacionados con la violencia de género. Por lo demás, y a fin de garantizar el derecho de las mujeres a recibir plena información, una asistencia integral total y un asesoramiento jurídico adecuado a su situación personal, se han desarrollado diversas políticas. Además de las campañas de comunicación, la secretaría General de Políticas de

${ }^{10}$ LÓPEZ PINA «Constitucioinalismo y religión civil, a modo de prólogo para españoles», LóPEz Pina, A. (ed), División de poderes e interpretación. Hacia una teoría praxis constitucional, Madrid: Tecnos, 1987. 
Igualdad ha editado más de 727.000 folletos y materiales divulgativos sobre los derechos de las mujeres víctimas en general o dirigidos a sectores específicos de la sociedad. Se ha creado también el servicio telefónico de información y asesoramiento jurídico a través del número 016 (900 116016 para personas con discapacidad auditiva). Por último, la ley preveía la inserción de esta igualdad en la educación y así en efecto se ha producido: no sólo mediante la puesta en práctica de planes de formación básica del profesorado, sino a través de la inserción en los planes de estudio de la asignatura de educación para la ciudadanía. La implantación en España por primera vez de una asignatura que explicara y difundiera los valores constitucionales, en efecto, no podía dejar de lado, uno de los principios básicos del constitucionalismo, la igualdad, y en concreto, la igualdad entre hombre y mujer.

\section{A MODO DE CONCLUSIONES: LA IGUALDAD COMO IMPERATIVO CONSTITUCIONAL.}

Con la implantación del Estado social de Derecho la igualdad ha cobrado un especial significado: igualdad que no sólo se proyecta como igualdad formal o igualdad ante la ley; la democratización del poder abre las puertas a reclamaciones de justicia material y así de igualdad sustantiva o material: igualdad de oportunidades que asegure que todos gocen de una igual libertad. Entre las proyecciones de esta igualdad se encuentra la igualdad entre hombre y mujer, la paridad electoral, en fin, la lucha contra la violencia de género.

La igualdad se configura en nuestra Carta Magna no sólo como un valor (artículo 1.1 CE), es asimismo un derecho fundamental (artículo $14 \mathrm{CE}$ ) y un principio político, que impone la actuación de los poderes públicos (artículo 9.2 CE). El artículo 1.1 CE consagra como valores fundamentales del orden jurídico la libertad, la justicia, la igualdad y el pluralismo político. La formulación liberal del principio de igualdad, recogida en el artículo $14 \mathrm{CE}$, como interdicción de toda diferenciación jurídico-formal, no parece suficiente para hacer realidad la dimensión social propia del vigente constitucionalismo. Justo por ello incorpora nuestro constituyente el artículo 9.2 CE; menciona expresamente el compromiso constitucional de los poderes públicos de asegurar una «igualdad real y efectiva», una igualdad material y no meramente formal. Supone reconocer la existencia de desigualdades reales y efectivas en nuestra sociedad, y la consiguiente directriz a los poderes públicos para combatirlas. 
Así, frente a quienes interpretan la igualdad como una exigencia de igualdad formal absoluta, hay autores que comprenden la igualdad «más en la línea de aceptar en ciertos supuestos el sacrificio (con límites, ciertamente) de la igualdad formal en aras de lograr mayores cotas de igualdad real» ${ }^{11}$. Pues bien, en esta disyuntiva, parece que la opción del constituyente de 1978 fue clara: no se configura la Constitución como una norma neutra. La cláusula del Estado social, determinante del nuevo constitucionalismo ${ }^{12}$, se incorpora a nuestra Carta Magna en su artículo primero. Irradia al resto de los preceptos constitucionales y explica la vocación transformadora de la Constitución ${ }^{13}$. Como observan López Pina y GuTIÉRREZ, «la igualdad formal vigente (la ley obliga a todos por igual) es superada así mediante la promoción de condiciones nuevas de igualdad material. Históricamente se observan funciones similares en la supresión de barreras para el acceso de todos a la propiedad inmueble cuando se desarticula el sistema feudal, o en la compensación de los desequilibrios derivados de la economía capitalista mediante la redistribución directa o indirecta cuando se supera el Estado liberal» ${ }^{14}$. En definitiva, optar por un Derecho neutro ante una situación de desigualdad real y efectiva, no es una decisión neutral. Supone aceptar el mantenimiento y la conservación de tal situación y desconocer, pues, la directriz constitucional reflejada en el artículo 9.2 CE. Este artículo, en definitiva, permite el diseño de un proyecto emancipador en el que el legislador, por propio imperativo constitucional, deviene protagonista. Y es que, en efecto, como proyecto ha de considerarse la remoción de las desigualdades que sustenta la ausencia de igualdad entre hombre y mujer, que hunde sus raíces en un modelo de sociedad del que seguramente todavía no nos hemos conseguido emancipar, al menos del todo. Ciertamente, las revoluciones liberales surgidas al calor de la Ilustración pudieron sustituir un modelo basado en el poder divino de los reyes por un orden asentado en un modelo racional de legitimación. Pero la reivindicación de igualdad que surge de aquí no se proyecta con igual intensidad en todos los ámbitos. Por lo demás, la pretensión de normatividad de los postulados ilustrados sólo con dificultades

${ }^{11}$ MARTín VIDA, M ${ }^{\mathrm{a}}$ A, Fundamento y límite constitucionales de las medidas de acción positiva, Cívitas, Madrid, 2002, pág. 109

${ }^{12}$ CABO, C. de, La crisis del Estado social, PPU, Barcelona, 1986, pág. 17

${ }^{13}$ Garronea Morales, A, El Estado español como Estado social y democrático de Derecho, Tecnos, Madrid, 1987, págs. 48 y ss.

${ }^{14}$ López Pina, A; Gutiérrez Gutiérrez, I, Elementos de Derecho Público, Marcial Pons, Madrid, 2002, pág. 34 
ha podido proyectarse también en el ámbito privado, en especial, en el de las relaciones afectivas. Ello ha incidido con especial intensidad en las posibilidades de asegurar una igualdad real entre hombre y mujer.

Para analizar la pertinencia de las políticas promocionales de la mujer hemos de acudir por tanto, como observa Cámara Villar, a la «caracterización del Estado español como social y democrático de Derecho (artículo 1.1.CE), debiéndose hacer hincapié en el calificativo social, que expresa un principio teleológico, político, de acción, que, entre otros fines y objetivos, persigue eliminar o allanar los obstáculos que impidan la efectividad de la igualdad y el de velar por las personas y grupos que de manera transitoria o permanente estén en situación de inferioridad vital. La Constitución española es, en esta dimensión, sustancialmente transformadora ${ }^{15}$. Esta dimensión emancipadora de la Constitución española debe proyectarse en diferentes aspectos; también en la promoción del sexo femenino como perteneciente a un grupo históricamente discriminado.

La violencia de género ha llegado a ser en los últimos tiempos asunto central para el foro público, la realidad social que aborda la ley orgánica evidencia claras desigualdades; las estadísticas así lo avalan. Ahora bien, la potestad por parte del legislador para hacer clasificaciones y discriminaciones no puede ser ilimitado en un Estado de Derecho; el límite no sólo lo encuentra en el resto de los derechos fundamentales, sino precisamente en las cláusulas expresas de proscripción de discriminación del artículo 14 CE. Giménez Glück, al analizar la jurisprudencia del Tribunal Constitucional sobre el principio de igualdad (artículo $14 \mathrm{CE}$ ), distingue entre un juicio de mínimos, que es el juicio ordinario de igualdad, y un juicio estricto. Aquél debe dejar un amplio margen de actuación al legislador: sólo se pueden declarar inconstitucionales las arbitrariedades flagrantes y manifiestas. El juicio estricto debe aplicarse a aquellas regulaciones normativas que utilicen como criterios alguno de los parámetros enunciados en el artículo 14 CE o cuando limiten derechos fundamentales ${ }^{16}$. En este caso, el juicio ha de ser mucho más severo; procesalmente, destaca la inversión de la carga de la prueba: la existencia de indicios razonables de que la diferenciación está perjudicando al

\footnotetext{
${ }^{15}$ CÁmara Villar, »Prólogo», Martín VIDA, M ${ }^{\mathrm{a}}$ A, Fundamento y límites constitucionales de las medidas de acción positiva, Cívitas, Madrid, 2002, pág. 15

${ }^{16}$ Giménez GLÜCK, D, Juicio de igualdad y Tribunal Constitucional, Bosch, Madrid, 2004, pág. 121
} 
colectivo marginado hace que sea el legislador el que deba probar la condición no discriminatoria de la medida adoptada ${ }^{17}$.

Pues bien, cabe plantearse qué tipo de juicio debe realizarse ante medidas de acción o discriminanción positiva. De nuevo nos encontramos ante diversas sensibilidades al interpretar la virtualidad de las cláusulas del artículo $14 \mathrm{CE}$. Para cierta doctrina, las disposiciones del mencionado precepto imponen un endurecimiento del juicio de igualdad cualquiera que sea el colectivo perjudicado por el uso del criterio discriminatorio. Otra parte de la doctrina entiende que los criterios del artículo $14 \mathrm{CE}$ vedan su utilización para perjudicar a determinados colectivos históricamente marginados; en cambio, si se trata de beneficiarlos, el juicio al que debe verse sometida tal medida debe ser un juicio de mínimos. Giménez Glück postula la creación de un juicio intermedio para las normas que benefician a determinados colectivos marginados. El relativo endurecimiento del test de igualdad se ha de ver aplacado por el mandato de igualdad del artículo 9.2 $\mathrm{CE}^{18}$. Este juicio intermedio, que implica un juicio más severo que el mero atender exclusivamente a las desproporciones manifiestas, parece pertinente cuando el control se realiza en un campo especialmente sensible como el ámbito del Derecho Penal. Ciertamente, el artículo 9.2 CE podría justificar el trato favorable al colectivo desfavorecido; la igualdad material se constituye en finalidad materialmente impuesta.

${ }^{17}$ Giménez GlücK, D, Juicio de igualdad y Tribunal Constitucional, Bosch, Madrid, 2004, pág. 301

${ }^{18}$ Giménez GlücK, D, Juicio de igualdad y Tribunal Constitucional, Bosch, Madrid, 2004, pág. 121 